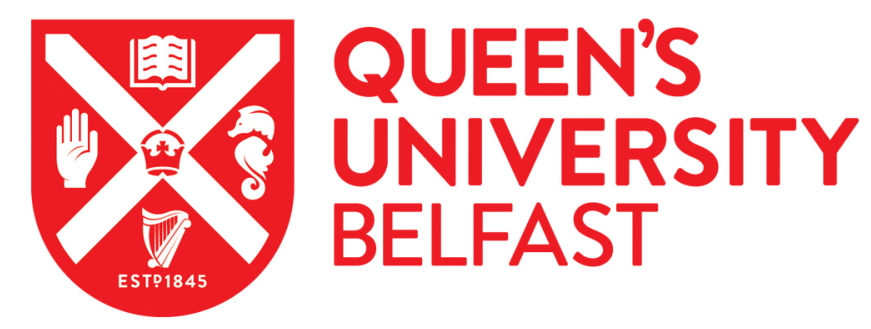

\title{
Human Herpesvirus 8 Seroprevalence Among Children and Adolescents in the United States.
}

Anderson, L., Yan, L., Graubard, B. I., Whitby, D., Mbsia, G., Tan, S., Goedert, J. J., \& Engels, E. A. (2008). Human Herpesvirus 8 Seroprevalence Among Children and Adolescents in the United States. The Pediatric Infectious Disease Journal, 27(7)(7), 661-664. https://doi.org/10.1097/INF.0b013e3181691740

\section{Published in:}

The Pediatric Infectious Disease Journal

Queen's University Belfast - Research Portal:

Link to publication record in Queen's University Belfast Research Portal

\section{General rights}

Copyright for the publications made accessible via the Queen's University Belfast Research Portal is retained by the author(s) and / or other copyright owners and it is a condition of accessing these publications that users recognise and abide by the legal requirements associated with these rights.

Take down policy

The Research Portal is Queen's institutional repository that provides access to Queen's research output. Every effort has been made to ensure that content in the Research Portal does not infringe any person's rights, or applicable UK laws. If you discover content in the Research Portal that you believe breaches copyright or violates any law, please contact openaccess@qub.ac.uk. 
TABLE 1. Cost Effectiveness Analysis in Different Risk Groups

\begin{tabular}{lccrc}
\hline \hline \multicolumn{1}{c}{ Risk Group } & $\begin{array}{c}\text { Hospitalization } \\
\text { Risk Without PVZ }\end{array}$ & $\begin{array}{l}\text { Hospitalization } \\
\text { Risk With PVZ }\end{array}$ & NNT & C/E Ratio \\
\hline Without BPD With Siblings & 0.28 & 0.06 & 4.5 & USD 13,198 \\
With BPD With Siblings & 0.36 & 0.21 & 6.6 & USD 21,152 \\
With BPD Without Siblings & 0.20 & 0.12 & 12.5 & USD 43,027 \\
Without BPD Without Siblings & 0.05 & 0.01 & 25 & USD 89,902 \\
\hline
\end{tabular}

$5 \mathrm{~kg}$ and no drug waste. Each vial of PVZ costs USD 1000 in Argentina. Health-care costs assuming PVZ prevention or no-prevention were compared. The effectiveness measure was reduction of hospitalization, according to the results from the IMpact Study.

One hundred eighty-four children met inclusion criteria, 4 were lost during follow-up and 21 were excluded because they could receive PVZ. Therefore, 159 children were enrolled in the study; of these, 41 patients $(26 \%)$ required hospitalization for RSV. Hospitalization rates varied between $18 \%$ and $38 \%$ related to the year.

Median age at admission was 6 months, $77 \%$ of patients were admitted between the winter months June and July, mean length of stay was 18 days and 14 patients (34\%) required mechanical ventilation for a mean of 16 days. Two patients died.

Birth weight, gestational age, intrauterine growth retardation, male gender, BPD, and need for home oxygen were not statistically different between infants who required or not RSV hospitalization. However, social risk analyses shows higher hospitalization rates for children of mothers with incomplete primary school and those with siblings under 10 years of age; besides, hospitalization risk increases as the number of siblings does: with 3 or more household under 10 years of age the odds ratio was 4.5 (95\% CI: 1.8-12). In multivariate analyses we found that the odds ratio for hospitalization was more than 2-fold if there were household under 10 years of age (OR: 2.6; 95\% CI: 1.3-6.5). Inadequate maternal education (OR: $2.1 ; 95 \%$ CI: $0.9-4.9$ ) and presence of BPD (OR: 2.2; 95\% CI: 1-5.5) showed a trend towards higher hospitalization rates. Birth weight under $1000 \mathrm{~g}$ did not increase risk of admission in our population (OR: $0.6 ; 95 \%$ CI: 0.3-1.4)

Based on these data, the cost-effectiveness analysis was performed, considering 4 risk groups: (1) patients without BPD with siblings under 10 years of age; (2) patients with BPD with siblings under 10 years of age; (3) patients with BPD without siblings under 10 years of age; and (4) patients without BPD without siblings under 10 years of age. Expected hospitalization rates and hypothetical reduction were calculated for each group. Table 1 shows data for each group: hospitalization risks with and without PVZ, number needed to treat (NNT) and cost effectiveness, reflecting amount of money needed to prevent 1 hospitalization. If results from IMpact study are used in our group of patients without BPD with siblings under 10 years of age, a $78 \%$ reduction in hospitalization rate is to be expected, meaning USD 13,198 to avoid 1 hospitalization (NNT = 4.5). For the group with BPD with siblings under 10 years of age, a $39 \%$ reduction in hospitalization rate is to be expected, and figure would be USD 21,152 (NNT = 6.6).

The purpose of a cost-effectiveness analysis in health care is to help decision-makers determine how to allocate resources among a number of competing needs to maximize health outcomes from a limited budget. In Argentina, health economic evaluations have been recently developed through governmental and nongovernmental organizations that perform these analyses upon request for introduction of new high-cost technologies. Availability for these evaluations is related with special programs and at present times definition thresholds for cost effective and socially accepted technologies for Argentina are still lacking. In our country, PVZ introduction has generated controversies. Data from this study allow us to confirm that there is a high hospitalization rate $(26 \%)$ because of RSVrelated disease in the high-risk population described and that hospitalized infants show severe clinical condition and a large proportion requires mechanical ventilation and prolonged hospitalization. Besides, social factors, namely number of siblings, are strongly associated with serious infection by RSV, and therefore, biologic risk factors currently used in developed countries are not enough to define the risks for RSV hospitalization in low socioeconomic high risk groups in developing nations like Argentina. We speculate that prospective studies including socioeconomic variables may provide more information to develop better grounded recommendations for prophylaxis with high-cost drugs in underdeveloped countries.

\section{REFERENCES}

1. Bauer G, Aguerre V, Fariña D, et al. Respiratory course and predictive factors for respiratory morbidity in infants who had bronchopulmonary dysplasia. Pediatr Res. 2000;47:1786.

2. Klein ML, Coviello S, Bauer G, et al. The impact of infection with human metapneumovirus and other respiratory viruses in young infant and children at high risk for severe pulmonary disease. $J$ Infect Dis. 2006;193:1544-1551

3. Bauer G, Dussel V, Fariña D, et al. Infección por virus sincicial respiratorio en poblaciones vulnerables: riesgo biológico vs riesgo social. Arch Argent Pediatr. 2005;103:198-204.

4. The Impact-RSV Study Group. Palivizumab. A humanized respiratory syncytial virus monoclonal antibody, reduces hospitalization from respiratory syncytial virus infection in high-risk infants. Pediatrics. 1998; 102:531-537.

5. American Academy of Pediatrics. Revised indication for the use of Palivizumab and respiratory syncytial virus inmune globulin intravenous for the prevention of respiratory syncytial virus infection. Pediatrics. 2003;112:1442-1446

6. Detsky A, Laupacis A. Relevance of cost-effectivenes analysis to clinicians and policy makers. JAMA. 2007;298:221-224.

7. Fariña D, Rodriguez S, Bauer G, et al. Respiratory syncytial virus prophylaxis: cost-effective analysis in Argentina. Pediatr Infect Dis J. 2002;21:287-291.

8. Kamal-Bahl S, Doshi J, Campbell J. Economic analyses of respiratory syncytial virus immunoprophylaxis in high-risk infants: a systematic review. Arch Pediatr Adolesc Med. 2002;156:1034-1041.

9. Nuijten M, Wittenberg W, Lebmeier. Cost effectiveness of palivizumab for respiratory syncytial virus prophylaxis in high risk children: a UK analysis. Pharmacoeconomics. 2007;25:55-71.

10. Simoes EA. Environmental and demographic risk factors for respiratory syncytial virus lower respiratory tract disease. $J$ Pediatr. 2003;143(5 Suppl):S118-S126.

\section{HUMAN HERPESVIRUS 8 SEROPREVALENCE AMONG CHILDREN AND ADOLESCENTS IN THE UNITED STATES}

Lesley A. Anderson, PhD, *广 Yan Li, PhD, + Barry I. Graubard, PhD, + Denise Whitby, PhD, $\S$ Georgina Mbisa, BSc,, Sylvia Tan, MS, $ף$

James J. Goedert, MD, * and Eric A. Engels, MD* 


\begin{abstract}
We measured human herpesvirus-8 antibodies (K8.1 and orf73 enzyme immunoassays) in 4166 children, aged $6-17$ years, in a U.S. cross-sectional survey. Forty-six were K8.1 seropositive (weighted seroprevalence: $1.1 \%$ ) and 20 were orf 73 seropositive (weighted seroprevalence $0.4 \%$ ). K8.1 seropositivity was associated with asthma (odds ratio: 6.3; 95\% confidence interval: $2.4-16.9$ ) and hay fever $(3.5 ; 1.1-11.0)$, and there were borderline associations with measures of crowding and low socioeconomic status.
\end{abstract}

Key Words: human herpesvirus 8, HHV-8, Kaposi sarcomaassociated herpes virus, KSHV, children, adolescents, U.S., asthma, hay fever, socioeconomic status, familial clustering Accepted for publication January 16, 2008.

From the *Infections and Immunoepidemiology Branch, Division of Cancer Epidemiology and Genetics, National Cancer Institute, Rockville; †Cancer Prevention Fellowship Program, Office of Preventive Oncology, Division of Cancer Prevention, National Cancer Institute, National Institutes of Health, Bethesda; $\$$ Biostatistics Branch, Division of Cancer Epidemiology and Genetics, National Cancer Institute, Rockville; §Viral Epidemiology Section, AIDS Vaccine Program, SAIC-Frederick, NCI Frederick, Frederick; and $₫$ RTI International, Rockville, MD.

Supported in part by grants from the Intramural Research Program of the National Cancer Institute N01-CO-12400.

The Research and Development Office, Northern Ireland sponsored Dr Lesley Anderson to participate in the Cancer Prevention Fellowship Program, Office of Preventive Oncology, Division of Cancer Prevention, National Cancer Institute.

The content of this publication does not necessarily reflect the views or policies of the Department of Health and Human Services, nor does mention of trade names, commercial products, or organizations imply endorsement by the U.S. Government.

Address for correspondence: Eric A. Engels, MD, Infections and Immunoepidemiology Branch, Division of Cancer Epidemiology and Genetics, National Cancer Institute, 6120 Executive Blvd, EPS 7076, Rockville, MD 20892. E-mail: engelse@mail.nih.gov.

DOI: $10.1097 / \mathrm{INF} .0 \mathrm{~b} 013 \mathrm{e} 3181691740$

$\mathrm{H}$ uman herpesvirus 8 (HHV-8) is associated with the development of Kaposi sarcoma, primary effusion lymphoma, and multicentric Castleman disease. HHV-8 seroprevalence varies by geographic location but is low in most developed countries. Among adults in the general U.S. population, HHV-8 seroprevalence has been estimated to range from $2 \%$ to $7 \% .^{1,2}$ In the United States, HHV-8 infection is thought to mainly occur in adulthood and is associated with high-risk sexual behaviors. ${ }^{2}$

HHV-8 infection among children has been less well studied. Exposure to saliva seems to be the main mode of HHV-8 acquisition, and familial clustering has been reported in Africa. ${ }^{3}$ In Africa, HHV-8 seroprevalence in children is high and has been associated with low socioeconomic status. ${ }^{4}$ A study in South Texas reported high HHV-8 seroprevalence (26\%) among 123 healthy children aged $4-13$ years. ${ }^{5}$ Other studies investigating HHV-8 seropositivity were limited to U.S. children and adolescents infected with, or at high risk of infection with, human immunodeficiency virus ${ }^{6,7} \mathrm{We}$ used sera and data from the third National Health and Nutrition Examination Survey (NHANES III) to study HHV-8 epidemiology in a large representative sample of U.S. children and adolescents.

\section{METHODS}

NHANES III, conducted by the National Center for Health Statistics during 1988-1994, is a cross-sectional survey of the U.S. noninstitutionalized civilian population. Household interviews, standardized physical examinations, and biologic samples were collected. ${ }^{8}$ NHANES III included 39,365 individuals randomly sampled through a complex, multistage probability design. Non-Hispanic blacks and
Mexican Americans were oversampled. Of the 7368 children and adolescents aged 6-17 years enrolled in NHANES III, 6942 were examined and 5771 had phlebotomy performed. The present report is based on the 4166 subjects aged 6-17 years for whom surplus serum was available for testing.

HHV-8 antibody testing was performed during a period of 6 months using enzyme immunoassays (EIAs) to detect IgG antibodies to the HHV-8 K8.1 structural glycoprotein, expressed during lytic replication, and the orf73 protein (also known as LANA), expressed during latent infection. ${ }^{2}$ To account for plate-to-plate variability, we adjusted optical density (OD) measurements for samples by regressing them against the mean OD of the positive controls on the respective EIA plate. ${ }^{2}$ Cutoffs were based on the distribution of the residuals from the regression models, and were chosen to be high to increase the specificity and positive predictive value of the EIA results (termed "conservative cutoffs" throughout). As a consequence, we may have underestimated the prevalence of HHV-8 infection in children and adolescents in the United States. In a sensitivity analysis, we used previously published cutoffs (termed "alternative cutoffs"). ${ }^{2}$ These alternative cutoffs yielded higher seroprevalence estimates but did not fully account for plate-to-plate variability in the EIA results.

For each EIA, HHV-8 seroprevalence was calculated using the conservative and the alternative cutoff to provide a range. To compare seroprevalence in subjects differing by demographic variables and medical history, we present odds ratios (ORs) and 95\% confidence intervals (CIs) based on logistic regression. Individuals included in this report were older than the NHANES III children and adolescents who lacked surplus serum $(P<0.001)$, and the 2 groups differed by race/ethnicity $(P<0.001)$. Because these differences could have introduced bias, we generated a new set of sample weights by poststratifying the standard NHANES III sample weights, which reflect NHANES III sampling probabilities, so that the new weighted totals matched corresponding totals for the U.S. population by age, gender, and race/ethnicity. All analyses used these new sample weights. SAS-callable SUDAAN, version 9.0.1 (Research Triangle Park, NC, 2005) was used for all analyses.

Children who were tested for HHV-8 antibodies were linked to other sampled household members in NHANES III who had an HHV-8 determination. We used this information to examine whether K8.1 seropositive children had other household members (children or adults ${ }^{2}$ ) who were also seropositive. No child who was orf73 seropositive had a family member who was also orf73 seropositive.

\section{RESULTS}

Using the conservative assay cutoff, 47 of the 4166 children and adolescents were K8.1 seropositive, yielding a weighted seroprevalence of $1.1 \%(95 \% \mathrm{CI}: 0.7-1.4 \%)$. Only 20 children and adolescents were orf73 seropositive according to the conservative cutoff (weighted seroprevalence: $0.4 \%$; $95 \%$ CI: $0.2-0.6 \%$ ). Five children tested seropositive for both K8.1 and orf73 $(\kappa=0.14)$. Using the alternative cutoffs, K8.1 seroprevalence and orf73 seroprevalence increased to $5.7 \%$ (95\% CI: $4.9-6.3 \%)$ and $6.1 \%(95 \%$ CI: $5.3-6.3 \%)$, respectively.

With the conservative cutoff, K8.1 seroprevalence did not vary by gender, age, or race/ethnicity (Table 1). K8.1 seroprevalence was approximately 2-fold higher, albeit not statistically significant, among children and adolescents in households with fewer than 6 rooms, more than 5 people, an unemployed reference person, or less than $\$ 10,000$ annual income (Table 1).

Children and adolescents with asthma or hay fever were significantly more likely to be K8.1 seropositive than individuals without these conditions (ORs: 6.3 and 3.5, respectively; Table 1). Adjusting for age, sex, race/ethnicity, household smoking, or socio- 
TABLE 1. Subject Characteristics and Associations With HHV-8 Seropositivity Among 4166 U.S. Children Using the Conservative Assay Cutoffs

\begin{tabular}{|c|c|c|c|c|c|c|c|}
\hline \multirow[b]{2}{*}{ Characteristic } & \multirow[b]{2}{*}{ Total } & \multicolumn{2}{|c|}{ K8.1 Enzyme Immunoassay } & \multicolumn{2}{|c|}{ Orf73 Enzyme Immunoassay } & \multicolumn{2}{|c|}{ Either Enzyme Immunoassay } \\
\hline & & $\begin{array}{l}\text { No. Seropositive } \\
\text { (Weighted \%) }\end{array}$ & $\begin{array}{l}\text { Weighted OR } \\
\quad(95 \% \text { CI })\end{array}$ & $\begin{array}{l}\text { No. Seropositive } \\
\text { (Weighted \%) }\end{array}$ & $\begin{array}{l}\text { Weighted OR } \\
\quad(95 \% \text { CI })\end{array}$ & $\begin{array}{l}\text { No. Seropositive } \\
\text { (Weighted \%) }\end{array}$ & $\begin{array}{l}\text { Weighted OR } \\
\text { (95\% CI) }\end{array}$ \\
\hline \multicolumn{8}{|l|}{ Gender } \\
\hline Male & 2053 & $20(0.88)$ & 1.0 & $8(0.34)$ & 1.0 & $27(1.20)$ & 1.0 \\
\hline Female & 2113 & $27(1.33)$ & $1.5(0.6-4.0)$ & $12(0.44)$ & $1.3(0.5-3.6)$ & $35(1.63)$ & $1.4(0.6-3.0)$ \\
\hline \multicolumn{8}{|l|}{ Race/ethnicity } \\
\hline Non-hispanic white & 1024 & $10(1.4)$ & 1.0 & 5 & 1.0 & $15(1.41)$ & 1.0 \\
\hline Non-hispanic black & 1465 & $19(1.8)$ & $1.3(0.5-3.2)$ & 11 & $3.1(0.8-12.0)$ & $25(1.82)$ & $1.3(0.6-2.8)$ \\
\hline Mexican American & 1458 & $17(1.4)$ & $0.8(0.3-1.8)$ & 4 & $1.8(0.4-9.2)$ & $21(1.44)$ & $1.0(0.5-2.0)$ \\
\hline Other & 219 & $1(0.9)$ & $0.8(0.1-6.8)$ & 0 & - & $1(0.91)$ & $0.6(0.1-5.2)$ \\
\hline \multicolumn{8}{|l|}{ Age, yrs } \\
\hline $6-7$ & 747 & $7(1.18)$ & 1.0 & $2(0.37)$ & 1.0 & $9(1.55)$ & 1.0 \\
\hline $8-9$ & 780 & $8(0.31)$ & $0.3(0.1-1.1)$ & $3(0.29)$ & $0.8(0.1-6.2)$ & $11(0.61)$ & $0.4(0.1-1.4)$ \\
\hline $10-12$ & 1038 & $16(1.73)$ & $1.5(0.3-7.1)$ & $8(0.80)$ & $2.2(0.5-9.4)$ & $21(2.22)$ & $1.5(0.4-5.1)$ \\
\hline $13-17$ & 1601 & $16(1.03)$ & $0.9(0.2-3.2)$ & $7(0.22)$ & $0.6(0.1-3.7)$ & $21(1.22)$ & $0.8(0.3-2.3)$ \\
\hline \multicolumn{8}{|c|}{ Family reference person employed } \\
\hline Yes & 2937 & $28(0.87)$ & 1.0 & $11(0.29)$ & 1.0 & $38(2.41)$ & 1.0 \\
\hline No & 1043 & $18(1.97)$ & $2.3(0.7-7.8)$ & $7(0.56)$ & $1.9(0.5-7.8)$ & $22(1.15)$ & $2.4(1.1-5.1)$ \\
\hline \multicolumn{8}{|l|}{ Family yearly income } \\
\hline$\$ 0-\$ 9999$ & 803 & $15(1.70)$ & 1.0 & $4(0.28)$ & 1.0 & $16(1.76)$ & 1.0 \\
\hline$\$ 10,000-\$ 19,999$ & 1076 & $11(0.57)$ & $0.3(0.1-1.3)$ & $5(0.51)$ & $1.8(0.4-8.7)$ & $15(1.05)$ & $0.6(0.1-2.6)$ \\
\hline$\$ 20,000-\$ 44,999$ & 1435 & $14(1.38)$ & $0.8(0.3-2.4)$ & $3(0.27)$ & $1.0(0.2-4.9)$ & $17(1.65)$ & $0.9(0.3-2.6)$ \\
\hline$\geq \$ 50,000$ & 488 & $3(0.76)$ & $0.4(0.1-2.1)$ & $5(0.31)$ & $1.1(0.3-4.9)$ & $8(1.07)$ & $0.6(0.1-2.3)$ \\
\hline \multicolumn{8}{|l|}{ Family size } \\
\hline$<5$ people & 1840 & $14(0.74)$ & 1.0 & $11(0.44)$ & 1.0 & $24(1.16)$ & 1.0 \\
\hline$\geq 5$ people & 2326 & $33(1.52)$ & $2.1(0.8-5.8)$ & $9(0.33)$ & $0.8(0.2-2.8)$ & $38(1.69)$ & $1.5(0.6-3.4)$ \\
\hline \multicolumn{8}{|l|}{ Household size } \\
\hline$<5$ people & 1814 & $14(0.74)$ & 1.0 & $11(0.44)$ & 1.0 & $24(1.18)$ & 1.0 \\
\hline$\geq 5$ people & 2352 & $33(1.50)$ & $2.0(0.7-5.7)$ & $9(0.33)$ & $0.7(0.2-2.7)$ & $38(1.67)$ & $1.4(0.6-3.4)$ \\
\hline \multicolumn{8}{|l|}{ Rooms in house } \\
\hline$<6$ rooms & 2063 & $30(1.56)$ & 1.0 & $7(0.36)$ & 1.0 & $34(1.73)$ & 1.0 \\
\hline$\geq 6$ rooms & 2103 & $17(0.85)$ & $0.5(0.2-1.6)$ & $13(0.40)$ & $1.1(0.4-3.6)$ & $28(1.23)$ & $0.7(0.3-1.8)$ \\
\hline \multicolumn{8}{|l|}{ Household density } \\
\hline$<1$ person per room & 2313 & $19(0.74)$ & 1.0 & $14(0.44)$ & 1.0 & $31(1.18)$ & 1.0 \\
\hline$\geq 1$ person per room & 1853 & $28(1.50)$ & $1.4(0.5-4.3)$ & $6(0.33)$ & $0.8(0.2-3.6)$ & $31(1.67)$ & $1.1(0.4-2.9)$ \\
\hline \multicolumn{8}{|l|}{ Urbanization } \\
\hline Urban & 1966 & $24(1.12)$ & 1.0 & $8(0.19)$ & 1.0 & $30(1.29)$ & 1.0 \\
\hline Rural & 2200 & $23(1.08)$ & $1.0(0.3-2.9)$ & $12(0.55)$ & $2.9(0.9-8.9)$ & $32(1.51)$ & $1.2(0.5-3.0)$ \\
\hline \multicolumn{8}{|l|}{ Asthma } \\
\hline No & 3782 & $39(0.71)$ & 1.0 & $19(0.43)$ & 1.0 & $54(4.34)$ & 1.0 \\
\hline Yes & 383 & $8(4.34)$ & $6.3(2.4-16.9)$ & $1(0.07)$ & $0.2(0.0-1.4)$ & $8(1.06)$ & $4.3(1.6-11.2)$ \\
\hline \multicolumn{8}{|l|}{ Hay fever } \\
\hline No & 3922 & $40(0.90)$ & 1.0 & $17(0.36)$ & 1.0 & $53(1.18)$ & 1.0 \\
\hline Yes & 244 & $7(3.08)$ & $3.5(1.1-11.0)$ & $3(0.66)$ & $1.8(0.4-8.7)$ & $9(3.68)$ & $3.4(1.3-8.9)$ \\
\hline \multicolumn{8}{|l|}{$\mathrm{Cold} / \mathrm{flu}$ in past $12 \mathrm{mo}$} \\
\hline No & 908 & $7(0.56)$ & 1.0 & $3(0.35)$ & 1.0 & $9(0.86)$ & 1.0 \\
\hline Yes & 3258 & $40(1.23)$ & $2.2(0.5-9.8)$ & $17(0.40)$ & $1.1(0.2-6.5)$ & $53(1.54)$ & $1.7(0.8-3.9)$ \\
\hline \multicolumn{8}{|l|}{ Sinusitis in past 12 mo } \\
\hline No & 3465 & $39(0.93)$ & 1.0 & $16(0.34)$ & 1.0 & $52(2.04)$ & 1.0 \\
\hline Yes & 695 & $8(1.74)$ & $1.9(0.7-5.5)$ & $4(0.57)$ & $1.7(0.4-6.7)$ & $10(1.24)$ & $1.7(0.4-6.7)$ \\
\hline
\end{tabular}

Totals do not always sum to $\mathrm{n}=47 \mathrm{~K} 8.1$ seropositive or $\mathrm{n}=20$ orf73 seropositive subjects, or to $\mathrm{n}=4166$ for the total population, because of missing questionnaire data.

economic status variables did not alter these associations (data not shown). K8.1 seropositivity was higher among children and adolescents who had a cold/flu or sinusitis in the 12 months before the survey, although these relationships were not significant (Table 1). Among K8.1 seropositive subjects, K8.1 antibody concentration was significantly higher in the subset who had had sinusitis (mean OD: 2.50 versus $2.10 ; P=0.005$ ) and nonsignificantly higher in those who had had cold/flu (mean OD: 2.20 versus 1.97; $P=0.29$ ) in the preceding 12 months.

With the conservative cutoff, there were very few orf73 seropositive subjects and no associations were significant (Table 1). With the alternative cutoffs, K8.1 seroprevalence seemed to increase with increasing age ( $P$ for trend $=0.04$, data not presented). Only the association between asthma and K8.1 seropositivity remained statistically significant (OR: 2.4; 95\% CI: 1.2-4.9). When we considered individuals as seropositive if they were seropositive according to either assay, the results generally mirrored those using the K8.1 enzyme immunoassay alone (Table 1).

Of the $42 \mathrm{~K} 8.1$ seropositive children and adolescents who had a family member tested for K8.1 antibody, 4 (9.5\%) had at least 1 family member who was K8.1 seropositive. In comparison, of the 3677 K8.1 seronegative children and adolescents who had a family member tested, $166(4.5 \%)$ had a family member who was K8.1 seropositive.

\section{DISCUSSION}

This is the largest study to investigate HHV-8 epidemiology among children and adolescents in the general U.S. population. K8.1 seroprevalence was significantly higher in children with asthma, hay 
fever, and, possibly, sinusitis. Although not statistically significant, our analysis suggested that K8.1 seropositivity was also associated with lower socioeconomic circumstances.

The major limitation of this study, as for other studies of HHV-8 epidemiology, is the lack of standard serological assays for HHV-8 infection. Although we used established EIAs to detect IgG antibodies to 2 viral proteins, variability in the testing results over time required us to take a new approach in standardizing the results across assay plates. ${ }^{2}$ We expect that this approach reduced the assay variability and increased the reliability of comparisons between groups of subjects, but the sensitivity and specificity of our assays under this approach are unknown. The poor agreement between the 2 EIAs observed in this study $(\kappa=0.14)$ probably reflects the low prevalence of HHV-8 in the general U.S. population as well as the assay performance. In addition, we chose highly conservative cutoffs to increase the specificity of the EIAs, which may have caused us to underestimate HHV-8 seroprevalence. HHV-8 seroprevalence was higher using the alternative cutoffs, but these results would be expected to be more variable, less specific, and associated with a higher false positive rate than the conservative cutoffs.

Because the EIAs have uncertain sensitivity and specificity, we are reluctant to interpret our seroprevalence results as estimates of the true prevalence of HHV-8 infection among U.S. children and adolescents. As expected, K8.1 and orf 73 seroprevalence were lower than we previously reported for U.S. adults using these same assays, ${ }^{2}$ possibly explained, in part, by an increase in prevalence of HHV-8 with age. Our estimates were also much lower than that reported in predominantly Hispanic children aged 4-13 years in a study from South Texas, ${ }^{5}$ which could reflect differences in the study population or HHV-8 testing. We did not see any difference in HHV-8 seroprevalence between white and Mexican-American children or adolescents in our study. A limitation is that our findings represent HHV-8 seroprevalence between 1988 and 1994, and changes in HHV-8 epidemiology may have occurred during the last 14 years.

Evidence for associations with socioeconomic status and familial clustering have been demonstrated in studies from areas with high HHV-8 seroprevalence. ${ }^{3}$ Although the mode of HHV-8 transmission is unknown, virus can be detected in saliva, and it is plausible that poverty or crowded living conditions could facilitate HHV-8 transmission by this route. Although we found no significant associations, HHV-8 seroprevalence seemed to be higher in children with lower socioeconomic status. Our study also suggested that familial clustering can occur. However, our findings regarding familial clustering should be considered descriptive because the low seroprevalence of HHV-8 and the complexity of the survey design prevented us from conducting a formal statistical analysis.

This is the first study to report an association of HHV-8 seropositivity with asthma and hay fever. We also observed nonsignificantly higher seroprevalence in subjects who had had cold/flu or sinusitis in the previous 12 months. In a prospective study, 5 of 6 Egyptian children with primary HHV-8 infection presented with fever and skin rash, followed by a secondary upper or lower respiratory infection. ${ }^{9}$ We speculate that the association between K8.1 seropositivity and respiratory illnesses may be due, at least in part, to symptoms related to recent primary infection. However, these associations could possibly reflect increased vulnerability to HHV-8 infection among children with respiratory disease, or mere chance, because we tested multiple associations.

In conclusion, our study found suggestive evidence of household transmission of HHV-8 among U.S. children and adolescents, although the results were not statistically significant. The relationships of HHV-8 seropositivity with respiratory conditions and hay fever warrant additional evaluation.

\section{REFERENCES}

1. Pellett PE, Wright DJ, Engels EA, et al. Multicenter comparison of serologic assays and estimation of human herpesvirus 8 seroprevalence among US blood donors. Transfusion. 2003;43:1260-1268.

2. Engels EA, Atkinson JO, Graubard B, et al. Risk factors for human herpesvirus 8 infection among adults in the United States and evidence for sexual transmission. J Infect Dis. 2007;196:199-207.

3. Plancoulaine S, Abel L, Tregouet D, et al. Respective roles of serological status and blood specific antihuman herpesvirus 8 antibody levels in human herpesvirus 8 intrafamilial transmission in a highly endemic area. Cancer Res. 2004;64:8782-8787.

4. Mbulaiteye SM, Biggar RJ, Pfeiffer RM, et al. Water, socioeconomic factors, and human herpesvirus 8 infection in Ugandan children and their mothers. J Acquir Immune Defic Syndr. 2005;38:474-479.

5. Baillargeon J, Leach CT, Deng JH, Gao SJ, Jenson HB. High prevalence of human herpesvirus 8 (HHV-8) infection in south Texas children. J Med Virol. 2002;67:542-548.

6. Goedert JJ, Charurat M, Blattner WA, et al. Risk factors for Kaposi's sarcoma-associated herpesvirus infection among HIV-1-infected pregnant women in the USA. AIDS. 2003;17:425-433.

7. Casper C, Meier AS, Wald A, Morrow RA, Corey L, Moscicki AB. Human herpesvirus 8 infection among adolescents in the REACH cohort. Arch Pediatr Adolesc Med. 2006;160:937-942.

8. Centers for Disease Control and Prevention. Analytic and reporting guidelines: the third National Health and Nutrition Examination Survey, NHANES III (1988-1994). 2007.

9. Andreone P, Gramenzi A, Lorenzini S, et al. Posttransplantation lymphoproliferative disorders. Arch Intern Med. 2003;163:1997-2004.

\section{ANAPLASMA PHAGOCYTOPHILUM INFECTION IN A CHILD}

\author{
Anna Psaroulaki, PhD, * Maria Koliou, PhD, $\dagger$ \\ Dimosthenis Chochlakis, MD, * Ioannis Ioannou, BSc, $†$ \\ Stella Mazeri, BSc, $\S$ and Yannis Tselentis, PhD*
}

Abstract: This is the first case of Anaplasma phagocytophilum infection described in Cyprus. A 9-year-old girl was infected after a tick-bite. The infection was diagnosed by molecular-based detection of the bacterium in 2 blood samples. The polymerase chain reaction product was sequenced, revealing a novel strain of Anaplasma phagocytophilum.

Key Words: Anaplasma phagocytophilum, child, PCR

Additional material related to this article and only published online can be accessed on the Web by clicking on the "ArticlePlus" link either in the Table of Contents or at the top of the Abstract or HTML version of the article.

Accepted for publication January 22, 2008.

From the *Laboratory of Clinical Bacteriology, Parasitology, Zoonoses and Geographical Medicine, School of Medicine, Crete, Greece; †Department of Paediatrics, Archbishop Makarios Hospital, Nicosia, Cyprus; $\$$ Veterinary Services, Nicosia, Cyprus; and §The Royal (Dick) School of Veterinary Studies, University of Edinburgh, Edinburgh, UK.

Address for correspondence: Anna Psaroulaki, PhD, Laboratory of Clinical Bacteriology, Parasitology, Zoonoses and Geographical Medicine, Voutes, Heraklion, Crete 71100, Greece. E-mail: annapsa@med.uoc.gr.

DOI: $10.1097 /$ INF.0b013e31816a0606

A naplasmoses are tick-borne emerging zoonoses (caused by bacteria within the family Anaplasmataceae). The causative agents are maintained through enzootic cycles between ticks and animals. ${ }^{1}$ 